\title{
When Ischemic Heart Failure Complicated with Diabetes Mellitus
}

\author{
Yuchao Zhang, Shaoping Wang* \\ Department of Cardiology, Beijing Anzhen Hospital, Capital Medical University, Beijing Institute of Heart Lung and Blood Vessel Diseases, \\ Beijing, China
}

*Corresponding Author: Shaoping Wang, Department of Cardiology, Beijing Anzhen Hospital, Capital Medical University, No 2 Anzhen Road, Chaoyang District, Beijing, 100029 China

Received Date: September 24, 2021; Accepted Date: October 09, 2021; Published Date: October 14, 2021

Citation: Yuchao Zhang, Shaoping Wang. (2021) When Ischemic Heart Failure Complicated with Diabetes Mellitus J. Clinical Cardiology and Cardiovascular Interventions, 4(17); Doi:10.31579/2641-0419/226

Copyright: () 2021 Shaoping Wang, This is an open-access article distributed under the terms of the Creative Commons Attribution License, which permits unrestricted use, distribution, and reproduction in any medium, provided the original author and source are credited.

\begin{abstract}
:
Diabetes and heart failure are mutually reinforcing factors. The treatment strategy and prognosis for patients with both diabetes and heart failure are inconclusive. This article reviews the current situation of ischemic heart failure complicated with diabetes, the pathophysiological relationship between diabetes and heart failure, and disease management. Especially, we highlight the latest result from CRISIS (Coronary Revascularization in Patients with Ischemic Heart Failure and Prevention of Sudden Cardiac Death) study, which finds that diabetes associates with greater ejection fraction improvement after revascularization in patients with reduced ejection fraction. This result implies the indication for revascularization in patients with heart failure who present with DM.

Keywords: ejection fraction; diabetes mellitus; ischemic heart disease; revascularization
\end{abstract}

\section{Introduction}

There are many factors to promote the occurrence of coronary artery disease (CAD). When patients are diagnosed with CAD and left ventricular dysfunction, the formulation of further treatment strategies still depend on the judgment of the underlying disease. Revascularization therapy, including percutaneous coronary intervention (PCI) and coronary artery bypass grafting $(\mathrm{CABG})$, is considered to be an active treatment strategy for patients with CAD and left ventricular dysfunction. However, which type of patients could benefit more from active revascularization is still unclear.

In the world, about one third of people with diabetes mellitus (DM) suffer from cardiovascular disease. Cardiovascular disease has become the main cause of death of diabetic patients. [1] The cost of treating cardiovascular disease accounts for $20 \%$ to $49 \%$ of the total cost of DM treatments, which has become serious medical and economic problems. [2] With the increase of the prevalence of heart failure (HF), the cost of health care increases, especially in patients with DM. [3]

DM and HF were mutually contributing factors. Previous studies had shown that diabetic patients with poor glucose control were more likely to develop HF. [4] Hyperglycemia and hyperinsulinemia accelerated the process of atherosclerosis through vascular smooth muscle proliferation and inflammation. Impaired insulin signal transduction might lead to cardiac stiffness, hypertrophy and fibrosis, which increases the risk of new or recurrent HF. [5] In patients with DM complicated with left ventricular dysfunction, the energy supply of cardiomyocytes depended more on the oxidation of fatty acids, abnormal energy metabolism, lipid accumulation and other factors promote cardiac systolic dysfunction. [6] Pathophysiological changes such as endothelial dysfunction, inflammation and oxidative stress had been strengthened, which further aggravated the process of myocardial dysfunction and cardiomyocyte apoptosis, and finally worsen cardiac function. [7]

In contemporary large scale studies of $\mathrm{HF}, 41 \%$ of patients had DM and another $29 \%$ were in pre- mellitus, which was a huge and surprising fact. [8] In addition, cardiac insufficiency increased the risk of developing DM in the future. Although the exact mechanism had not been clarified, current studies had shown that higher systolic blood pressure, longer duration of HF, higher New York Heart Association functional class and diuretic treatment were predictors of DM in patients with HF. [4]

Data from Swedish Coronary Angiography and Angioplasty Registry (SCAAR) showed that DM was independently associated with higher mortality, regardless of whether HF was an ischemic cause or not (HR, 1.40; 95\% CI, 1.33-1.46 and HR, $1.30 ; 95 \%$ CI, 1.22-1.39, respectively). [9] Glucose control was closely related to the prognosis of patients with HF. Active lifestyle could improve insulin resistance and glucose level, and then improved the prognosis. [10] With the deepening of research, the genetic mechanism of diabetes diet therapy has also been revealed, laying a foundation for the whole course of disease management. [11,12] Although enhanced glucose control could reduce the long-term risk of diabetic microvascular events, HbA1c was not linearly related to the risk 
of death in patients with HF. Contemporary observational studies had shown that there was a U-shaped correlation between $\mathrm{HbAlc}$ and mortality, and the death rate was the lowest when HbA1c was controlled at $7 \% \sim 8 \%$. Appropriate relaxation of $\mathrm{HbA} 1 \mathrm{c}$ control target was recommended for elderly patients with widespread complications and short life expectancy. [4] In the selection of glucose-lowering medications special attention should be paid to the glucose-lowering effect and the benefit of cardiovascular events. Recently, sodium-glucose co-transporter 2 inhibitors recommended in the 2021 ESC guidelines for the treatment of DM patients with HF for reducing cardiovascular mortality and hospitalizations rates in patients with DM complicated with HF (Class of recommendation: I , Level of evidence: A). [13] Furthermore, in diabetic patients with ischemic left ventricular dysfunction, modulation of free fatty acids metabolism could give greater benefits of decreasing the incidence of angina attacks than in non-diabetic patients. [14] The addition of direct renin inhibitor aliskiren to standard therapy was associated with trends towards greater reduction in left ventricular size among diabetic compared with non-diabetic subjects who had history of myocardial infarction and ejection fraction $(\mathrm{EF}) \leq 45 \%$. [15]

In addition to drug therapy, invasive revascularization was an important part of the treatment strategy for patients with CAD and left ventricular dysfunction. Especially for patients with DM and CAD, restoring sufficient coronary blood flow can be beneficial to the recovery of myocardial function in these patients. After careful evaluation of coronary artery anatomy, comorbidities, life expectancy, and patient expectation, revascularization can be considered as an active strategy for improving outcomes in patients with DM and CAD. [13] In the current guidelines, $\mathrm{CABG}$ was recommended as a first-line revascularization strategy for $\mathrm{HF}$ patients with DM or multivessel disease. [13] However, there were no randomized controlled trials of PCI or CABG in patients with HF complicated with DM. Previous randomized controlled studies have shown that the long-term survival rate of patients with $\mathrm{EF} \leq 35 \%$ treated with medical treatment combined with $\mathrm{CABG}$ was better than that of patients treated with optimal medical treatment alone (HR, 0.84; 95\% CI, 0.73-0.97; $P=0.02)$. [14] Results from SCAAR showed that DM patients with ischemic HF benefit more from CABG than PCI (all-cause mortality: OR, $0.62 ; 95 \%$ CI, 0.41-0.96; $P=0.031)$. [16] A clinical study comparing PCI or CABG in the treatment of DM patients with left ventricular dysfunction showed that PCI was associated with higher postoperative major adverse cardiovascular and cerebrovascular events (for EF 35\% to 49\%: HR, 1.97; 95\% CI, 1.64-2.35; $\mathrm{P}<0.001$; for $\mathrm{EF}<35 \%$ : HR, $2.28 ; 95 \%$ CI, 1.79-2.90; $\mathrm{P}<0.001)$. [17] A recent network meta-analysis showed that CABG significantly reduced the incidence of mortality, cardiac death, myocardial infarction and repeated revascularization in patients with ischemic left ventricular dysfunction compared with PCI and medical treatment. However, when returning to important covariates, DM did not show a significant correlation with all-cause mortality after CABG. [18]

In addition to clinical events as the hard end point of results, the improvement of EF could also be regarded as one of the evaluation indexes of patients with HF. Successful revascularization therapy might improve long-term outcome by attenuating the ischemic state and reversing left ventricular remodeling for patients with ischemic HF. [14] EF improvement following revascularization might be associated with outcomes of CAD patients with left ventricular dysfunction. Recently, Wang et.al [19] reported a real-world study i.e., Coronary Revascularization in Patients with Ischemic Heart Failure and Prevention of Sudden Cardiac Death (CRISIS). All patients had an initial EF $\leq 40 \%$ who underwent either PCI or CABG. A total of 974 patients with repeated EF measurements after revascularization were included. DM was found as an independent correlate of greater $\mathrm{EF}$ improvement after revascularization (OR, 1.42; 95\% CI, 1.07-1.89; $\mathrm{P}=.014)$. Patients with $\mathrm{DM}$ had a greater extent of $\mathrm{EF}$ improvement after revascularization $(10.5 \pm 10.4 \%$ vs $8.1 \pm 11.2 \%$; $\mathrm{P}=.002)$ compared with non-diabetic patients. In EF improved group, diabetic patients had similar risk of overall (HR, 1.36; 95\% CI, 0.80-2.32; P=.257) and cardiovascular mortality (HR, 1.42; 95\% CI, 0.77-2.64; $\mathrm{P}=.262$ ) compared with nondiabetic patients. In contrast, in EF unimproved group, DM was associated with higher risk of both overall (HR, 1.46; 95\% CI, 1.02-2.08; $\mathrm{P}=.037)$ and cardiovascular death (HR, 1.48; 95\% CI, 1.02-2.22; $\mathrm{P}=.046)$. This indicated that greater EF improvement by revascularization in diabetic patients at least partially attenuated the impact of DM on adverse long-term outcomes. Therefore, this finding imply the indication for revascularization in patients with left ventricular dysfunction who present with DM

Finally, despite the prevalence of HF and DM, the optimal treatment strategy including medication and revascularization therapy for patients with ischemic heart failure complicated with DM is still uncertain. Large scale randomized controlled trials are needed to enrich evidence-based medicine.

\section{Declaration}

Funding: Not applicable.

Conflict of Interest/Competing interests: All authors declare that they have no conflict of interest.

Author Contribution: Yuchao Zhang wrote the original-draft. Shaoping Wang edited and approved the final version of the manuscript.

\section{References}

1. Einarson TR, Acs A, Ludwig C, et al. Prevalence of cardiovascular disease in type 2 diabetes: a systematic literature review of scientific evidence from across the world in 20072017[J]. Cardiovasc Diabetol, 2018, 17(1): 83.

2. Einarson TR, Acs A, Ludwig C, et al. Economic Burden of Cardiovascular Disease in Type 2 Diabetes: A Systematic Review [J]. Value Health, 2018, 21(7): 881-890.

3. Lesyuk W, Kriza C, Kolominsky-Rabas P. Cost-of-illness studies in heart failure: a systematic review 2004-2016[J]. BMC Cardiovasc Disord, 2018, 18(1): 74.

4. Dunlay SM, Givertz MM, Aguilar D, et al. Type 2 Diabetes Mellitus and Heart Failure, A Scientific Statement From the American Heart Association and Heart Failure Society of America[J]. J Card Fail, 2019, 25(8): 584-619.

5. Kodama S, Fujihara K, Horikawa C, et al. Diabetes mellitus and risk of new-onset and recurrent heart failure: a systematic review and meta-analysis[J]. ESC Heart Fail, 2020, 7(5): 21462174.

6. Jia G, Hill MA, Sowers JR. Diabetic Cardiomyopathy: An Update of Mechanisms Contributing to This Clinical Entity[J]. Circ Res, 2018, 122(4): 624-638.

7. Randhawa VK, Dhanvantari S, Connelly KA. How Diabetes and Heart Failure Modulate Each Other and Condition Management [J]. Can J Cardiol, 2021, 37(4): 595-608.

8. Dauriz M, Targher G, Temporelli PL, et al. Prognostic Impact of Diabetes and Prediabetes on Survival Outcomes in Patients With Chronic Heart Failure: A Post-Hoc Analysis of the GISSIHF (Gruppo Italiano per lo Studio della Sopravvivenza nella Insufficienza Cardiaca-Heart Failure) Trial[J]. J Am Heart Assoc, 2017, 6(7).

9. Johansson I, Dahlstrom U, Edner M, et al. Prognostic Implications of Type 2 Diabetes Mellitus in Ischemic and Nonischemic Heart Failure[J]. J Am Coll Cardiol, 2016, 68(13): 1404-1416.

10. American Diabetes A. 5. Facilitating Behavior Change and 
Well-being to Improve Health Outcomes: Standards of Medical Care in Diabetes-2020[J]. Diabetes Care, 2020, 43(Suppl 1): S48-S65.

11. Martins IJ. Anti-Aging Genes Improve Appetite Regulation and Reverse Cell Senescence and Apoptosis in Global Populations [J]. Advances in Aging Research, 2016, 05(01): 9-26.

12. Martins IJ. Single Gene Inactivation with Implications to Diabetes and Multiple Organ Dysfunction Syndrome [J]. Journal of Clinical Epigenetics, 2017, 03(03).

13. McDonagh TA, Metra M, Adamo M, et al. 2021 ESC Guidelines for the diagnosis and treatment of acute and chronic heart failure[J]. Eur Heart J, 2021.

14. Velazquez EJ, Lee KL, Jones RH, et al. Coronary-Artery Bypass Surgery in Patients with Ischemic Cardiomyopathy [J]. N Engl J Med, 2016, 374(16): 1511-1520.

15. Shah AM, Shin SH, Takeuchi M, et al. Left ventricular systolic and diastolic function, remodelling, and clinical outcomes among patients with diabetes following myocardial infarction and the influence of direct renin inhibition with aliskiren[J]. Eur
J Heart Fail, 2012, 14(2): 185-192.

16. Volz S, Redfors B, Angeras O, et al. Long-term mortality in patients with ischaemic heart failure revascularized with coronary artery bypass grafting or percutaneous coronary intervention: insights from the Swedish Coronary Angiography and Angioplasty Registry (SCAAR)[J]. Eur Heart J, 2021, 42(27): 2657-2664.

17. Nagendran J, Bozso SJ, Norris CM, et al. Coronary Artery Bypass Surgery Improves Outcomes in Patients with Diabetes and Left Ventricular Dysfunction [J]. J Am Coll Cardiol, 2018, 71(8): 819-827.

18. Gaudino M, Hameed I, Khan FM, et al. Treatment strategies in ischaemic left ventricular dysfunction: a network meta-analysis [J]. Eur J Cardiothorac Surg, 2020.

19. Wang S, Borah B, Cheng S, et al. Diabetes Associated With Greater Ejection Fraction Improvement After Revascularization in Patients With Reduced Ejection Fraction [J]. Front. Cardiovasc. Med, 2021.
This work is licensed under Creative Commons Attribution 4.0 License

\section{To Submit Your Article Click Here: Submit Manuscript}

DOI:10.31579/2641-0419/226
Ready to submit your research? Choose Auctores and benefit from:

$>$ fast, convenient online submission

$>$ rigorous peer review by experienced research in your field

$>$ rapid publication on acceptance

$>$ authors retain copyrights

$>$ unique DOI for all articles

$>$ immediate, unrestricted online access

At Auctores, research is always in progress.

Learn more https://auctoresonline.org/journals/clinical-cardiology-andcardiovascular-interventions 